\title{
Intraoperative combined color and fluorescent images-based sentinel node mapping in the porcine lung: Comparison of indocyanine green with or without albumin premixing
}

\author{
Yujin Oh, BS, ${ }^{a}$ Yu Hua Quan, MS, ${ }^{\mathrm{b}}$ Yeonho Choi, PhD, ${ }^{\text {a }}$ Choong Ki Kim, BS, ${ }^{a}$ Heezoo Kim, MD, PhD, ${ }^{c}$ \\ Hyun Koo Kim, MD, PhD, ${ }^{b}$ and Beop-Min Kim, $\mathrm{PhD}^{\mathrm{a}}$
}

\begin{abstract}
Objectives: We developed a multimodal optical imaging system for intraoperative visualization of sentinel lymph nodes (SLNs). This study is to validate our system by showing SLNs in the lung through combined optical color and fluorescent image with indocyanine green (ICG) and ICG with human serum albumin (HSA).
\end{abstract}

\begin{abstract}
Methods: Identical ICG concentrations of ICG only or ICG:HSA was injected into the rat footpad and porcine lung. Absolute amounts of the fluorescents were scaled on the basis of animal weights. The entire procedures were recorded using color and near-infrared (NIR) charge-coupled device (CCD) cameras simultaneously, and the 2 images were merged by real-time image processing software. All fluorescence intensity signals to background ratio (SBR) and retention rates at SLN for both fluorescents were estimated and compared.
\end{abstract}

Results: This newly developed intraoperative color and fluorescence optical imaging system successfully visualized the SLNs in animal experiments. The SLNs were identified $100 \%$ for both rat and pig under in vivo conditions. Real-time image processing software overcame the low signal of NIR fluorescence images. ICG and ICG:HSA provided no significantly different SBR in the SLN images for both rat thigh and pig lung.

Conclusions: The intraoperative optical imaging system enabled real-time image-guided surgery during SLN mapping in lung in an animal model. The ICG retention rate was similar to ICG:HSA. ICG alone can be useful for SLN imaging during lung cancer surgery. (J Thorac Cardiovasc Surg 2013;146:1509-15)

The technique of sentinel lymph node (SLN) biopsy is a minimally invasive method of identifying the patients at greater risk and facilitating the selective use of more aggressive surgical and systemic therapies to improve outcomes with little additional morbidity. ${ }^{1}$ It is currently regarded as the standard treatment method for malignant melanoma and breast cancer, and applications are expanding to other malignant diseases, including lung cancer.

\footnotetext{
From the Department of Biomedical Engineering, ${ }^{\text {a }}$ Korea University; the Department of Thoracic and Cardiovascular Surgery, ${ }^{\mathrm{b}}$ Korea University Guro Hospital, Korea University College of Medicine; and the Department of Anesthesiology and Pain Medicine, ${ }^{\mathrm{c}}$ Korea University Guro Hospital, Korea University College of Medicine, Seoul, Korea.

This work was supported by the National Research Foundation of Korea (NRF) grant funded by the Ministry of Education, Science and Technology (No. 2012012166) and by a grant of the Korean Health Technology R\&D Project, Ministry of Health \& Welfare, Republic of Korea (No. A121074).

Disclosures: Authors have nothing to disclose with regard to commercial support.

Financial disclosure: All intellectual property associated with the ICFIS is owned by the Korea University.

Y.O. and Y.H.Q. contributed equally to this work.

Received for publication Dec 13, 2012; revisions received Jan 19, 2013; accepted for publication Feb 14, 2013; available ahead of print March 25, 2013.

Address for reprints: Hyun Koo Kim, MD, PhD, Department of Thoracic and Cardiovascular Surgery, Korea University Guro Hospital, Korea University College of Medicine, 97 Guro-dongkil, Guro-ku, Seoul 152-703, Korea (E-mail: kimhyunkoo@korea.ac.kr); or Beop-Min Kim, PhD, Department of Biomedical Engineering, Korea University, Horim 119Hall, Jungnung-1-dong, Seongbuk-gu, Seoul, 136-703, Korea (E-mail: bmk515@korea.ac.kr).

$0022-5223 / \$ 36.00$

Copyright $(2013$ by The American Association for Thoracic Surgery

http://dx.doi.org/10.1016/j.jtcvs.2013.02.044
}

In 1999, Little and colleagues ${ }^{2}$ first demonstrated that the detection rate of SLN in vivo is less than $50 \%$ for patients with lung cancer when isosulfan blue dye is used owing to poor visibility, particularly in the presence of intrathoracic black-pigmented anthracotic lymph nodes. ${ }^{3,4}$ Since Liptay and colleagues ${ }^{5}$ reported radiotracer methods using technetium $99 \mathrm{~m}$ sulfur colloid with an $82 \%$ identification rate and a 95\% accuracy rate, radioisotopes have been used in most studies of SLN mapping in lung cancer. However, when the primary lesion is directly injected with radioactive tracer, its radioactivity is so strong that the radioactivity of the true SLNs is masked, ${ }^{6}$ especially SLNs that are located at the hilar area in lung cancer. This phenomenon (called shine-through phenomenon) makes in vivo identification of SLNs more difficult in lung cancer with its complex and aberrant patterns of lymphatic drainage. ${ }^{4}$ To overcome this drawback, the surgeons should be trained to measure carefully the radioactivity of each lymph node and endure a certain period of learning curve. For this reason, the Cancer and Leukemia Group B (CALGB) 140203 multi-institutional phase 2 trial investigating the intraoperative SLN mapping in lung cancer using radioisotope was unsuccessful, ${ }^{7}$ although many single-center trials including our studies showed successful results. ${ }^{8-12}$

Recently, the near-infrared (NIR) fluorescence imaging system has attracted considerable interest in the field of SLN identification. ${ }^{13}$ Optical imaging using the NIR 


$$
\begin{aligned}
\text { Abbreviations and Acronyms } \\
\text { CCD }=\text { charge-coupled device } \\
\text { HSA }=\text { human serum albumin } \\
\text { ICFIS }=\text { intraoperative color and fluorescence } \\
\quad \text { imaging system } \\
\text { ICG }=\text { indocyanine green } \\
\text { NIR }=\text { near-infrared } \\
\text { SBR }=\text { signal to background ratio } \\
\text { SLN }=\text { sentinel lymph node }
\end{aligned}
$$

fluorescent lymphatic tracer enables real-time visualization of lymphatic channels and SLNs during operation. ${ }^{14}$ Therefore, NIR fluorescence imaging could provide an alternative for, or an addition to, conventional techniques used for SLN mapping with several advantages such as better penetration and visibility than blue dyes and capability of real-time visualization and removing ionizing radiation over radiotracers. ${ }^{6,15}$

Indocyanine green (ICG) is the only clinically available NIR fluorescent lymphatic tracer. ${ }^{16}$ Even if the dye itself is not visible in anthracotic lymph nodes ${ }^{17}$ its fluorescence is in the NIR region and can be easily detected with an infrared camera. However, it is said that ICG has some problems as an optimal tracer: relatively low fluorescence brightness and small hydrodynamic diameter, which can lead it to flow to higher-tier lymph nodes with poor retention in the SLN. ${ }^{14}$ Ohnishi and colleagues ${ }^{18}$ have demonstrated that adsorption of ICG to human serum albumin (HSA), by simply premixing ICG with HSA, can increase the fluorescence intensity and the hydrodynamic diameter, giving it better SLN retention with an improved detection rate over ICG alone in the preclinical trials.

We developed an optical imaging system, called intraoperative color and fluorescence imaging system (ICFIS), that provides color and fluorescence images of the target tissues along with their fused images. It allows the surgeon to identify SLNs intraoperatively within the context of surgical anatomy. In this study, before clinical application of the ICFIS in SLN mapping in lung cancer, we evaluated the clinical usefulness of 2 fluorescents including ICG and ICG:HSA in animal models.

\section{MATERIALS AND METHODS Intraoperative Color and Fluorescence Imaging System}

The ICFIS sits on a custom-manufactured $105 \mathrm{H} \times 60 \mathrm{D} \times 120 \mathrm{H} \mathrm{cm}$ moving cart with 4 rotating and locking wheels. An articulating arm boom stand with 780 -mm maximum extension and $\pm 210 \mathrm{~mm}$ articulating range firmly holds the system (Figure 1, A).

An excitation beam from a 750-nm diode laser up to $1.2 \mathrm{~W}$ (SDL-750LM-XXXT; Shanghai DLT Co, Ltd, Shanghai, China) is delivered to the region of interest via a 2-m long flexible bifurcated light guide. Beam intensities were measured at $10 \mathrm{~mW} / \mathrm{cm}^{2}$ for rat and $5 \mathrm{~mW} / \mathrm{cm}^{2}$ for pig, each of which was optimized on the basis of the injected volume of the fluorophore.

Both reflected white light and fluorescence are collected with a zoom lens. The working distance between the zoom lens and object is set to 60 $\mathrm{cm}$. The surgical field of view is set to $40 \mathrm{~W} \times 30 \mathrm{H} \mathrm{cm}$ (Figure $1, B$ ). The white light and fluorescence are separated by a dichroic mirror with a cutoff frequency of $675 \mathrm{~nm}$. The fluorescence reflected off the dichroic mirror passes through a 1-inch long wavelength pass filter at $808 \mathrm{~nm}$ before reaching a NIR charge-coupled device (CCD) camera (CM-141MCL; JAI Ltd, Yokohama, Japan). Reflected light that is transmitted through the dichroic mirror is further filtered via another 1-inch short-wavelength pass filter at $650 \mathrm{~nm}$ and detected by a color CCD camera (IMC-15FT; IMI-tech, Anyang, Korea) (Figure 1, C).

Each camera produces images at different frames per second (30 for NIR CCD camera and 15 for color CCD camera). Only the NIR images were processed using software so that only the signal over a certain threshold is captured, which is later overlaid on top of the color image during realtime visualization.

\section{Preparation of ICG and ICG:HSA}

ICG (25-mg vials) was purchased from Jeil Pham (Daiichi-Sankyo Korea Co, Ltd; Seoul, Korea) and was dissolved in $10 \mathrm{~mL}$ of saline solution to yield a $2.5-\mathrm{mg} / \mathrm{mL}$ (3.2-mmol/L) stock solution. ICG, 10, 20, 40, and 80 $\mu \mathrm{L}$, was mixed with $1.25,2.5,5,10 \mu \mathrm{L}$, respectively, of $20 \%$ HSA solution (SK Chemical, Seoul, Korea) to yield ICG in HSA (ICG:HSA) at various concentrations $(3.2,6.4,12.8$, and $32 \mu \mathrm{mol} / \mathrm{L})$.

\section{Animal Preparation}

Five-week-old female rats (SK Chemical, Seoul, Korea) of $200 \mathrm{~g}$ were used for in vivo experiments. They were administered $20 \mathrm{mg} / \mathrm{kg}$ of zolazepam plus tiletamine (Zoletil; Vairbac, Carros, France) intramuscularly, and either ICG or ICG:HSA was injected into the footpad. Each group comprised 5 animals. At each different time after injection, the ipsilateral leg was exposed from foot to inguinal area by skin incision. Lymphatic flow of this area was observed in real time using ICFIS, and the first lymph node was identified and removed.

Female Yorkshire pigs (Yokenongzang; Yeonchen, Korea) weighing a mean of $30 \mathrm{~kg}$ had anesthesia induced with $10-\mathrm{mg} / \mathrm{kg}$ intramuscular xylazine (Rompun; Bayerkorea, Seoul, Korea). A mechanical ventilator was connected to the tracheostomy site and anesthesia was maintained with 2\% isoflurane (Baxter Healthcare, Deerfield, Ill). The vital signs were monitored throughout the operation by a clinical anesthetist. Unilateral chest wall resection was performed from the second intercostal space to the diaphragm level to obtain the whole image of the thoracic cavity including lung and mediastinal structures (Figure 2). Resection could be done with conventional devices such as a rib cutter and electrocauterization. Pulmonary lymphatic mapping was performed by injecting ICG or ICG:HSA intraparenchymally using a tuberculin syringe. Each group comprised 2 animals. Injections were positioned approximately $1-\mathrm{cm}$ deep into the lateral surface of the left upper lobes. Lymphatic flow was visualized in real time using ICFIS. The first lymph node encountered on the path delineated by the fluorescent lymphatics was defined as the SLN. All procedures were approved by the Korea University Medical Center's Animal Experimentation Committee and were carried out in accordance with the institutional guidelines for the care and use of laboratory animals.

\section{Statistical Analysis}

For comparing the signal to background ratio (SBR) of in vivo SLN in rat between ICG and ICG:HSA, Wilcoxon rank-sum test was performed. Null hypotheses of no difference were rejected if $P$ values were less than .05. Statistical software (SPSS for Windows, version 12.0; SPSS, Inc, Chicago, Ill) was used for statistical analysis. 

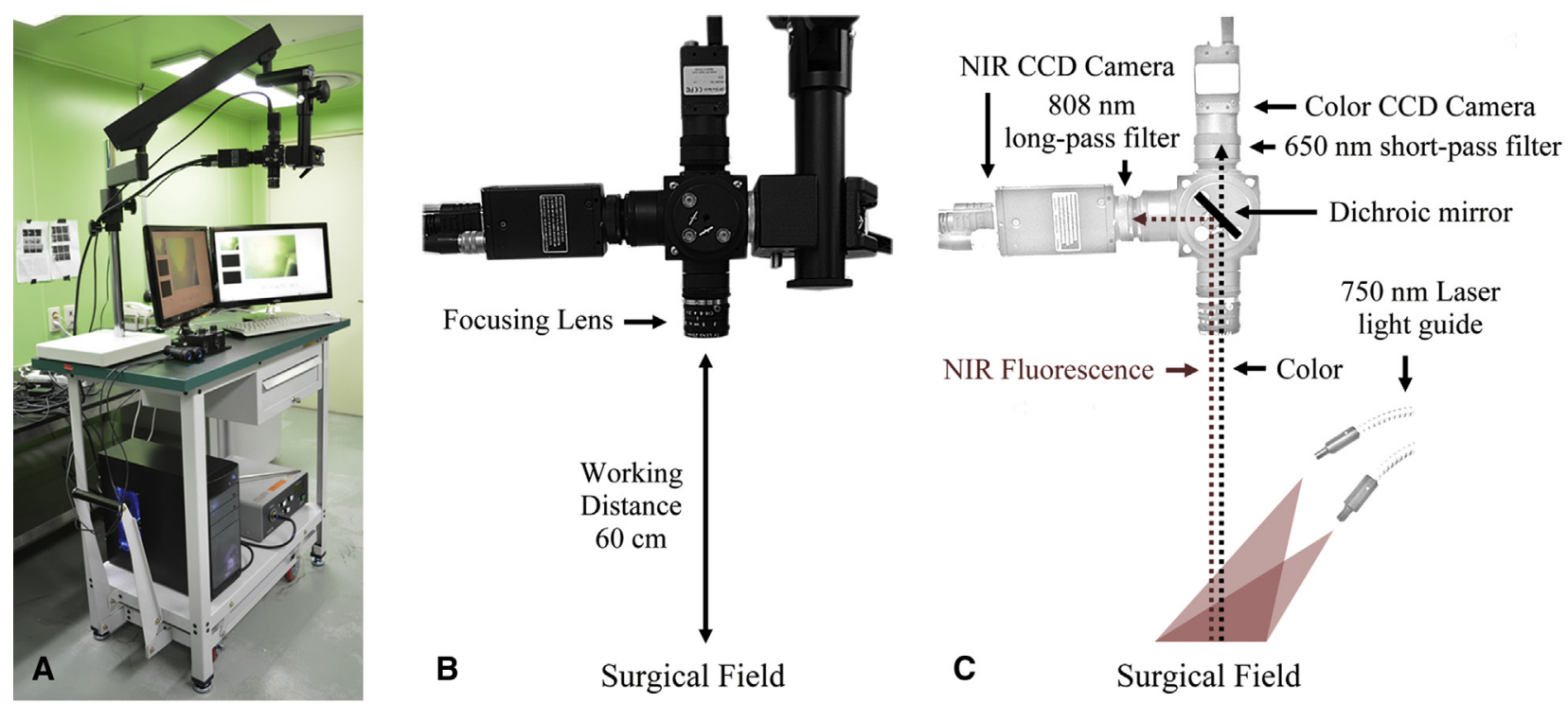

FIGURE 1. Intraoperative color and fluorescence imaging system. A, System mounted on a moving cart. B, Magnified image of the optics and camera arrays. The working distance between the zoom lens and object was set to $60 \mathrm{~cm}$ and the field of view was set to $40 \mathrm{~cm} \mathrm{~W} \times 30 \mathrm{~cm} H$ to cover the entire parts of the lung. C, Details of parts shown in B. Illustration of light paths of color and fluorescence light. NIR, Near-infrared; $C C D$, charge-coupled device.

\section{RESULTS}

\section{Intraoperative Color and Fluorescence Imaging System}

ICFIS successfully visualized the NIR fluorescence from all SLNs during the entire surgical procedure under the ordinary bright surgical environment. Inasmuch as NIR light is invisible to the human eye, the surgical view was not disturbed by NIR excitation light nor by the NIR fluorescence of SLN tracers. Inasmuch as the long wavelength portion of the bright room light slightly contaminates the fluorescence image, the contrast of the NIR fluorescence images was manually controlled using software depending on the surgical field conditions. Modulated NIR fluorescence images were then pseudocolored, overlaid on the color images, and finally displayed on the monitors in real time.

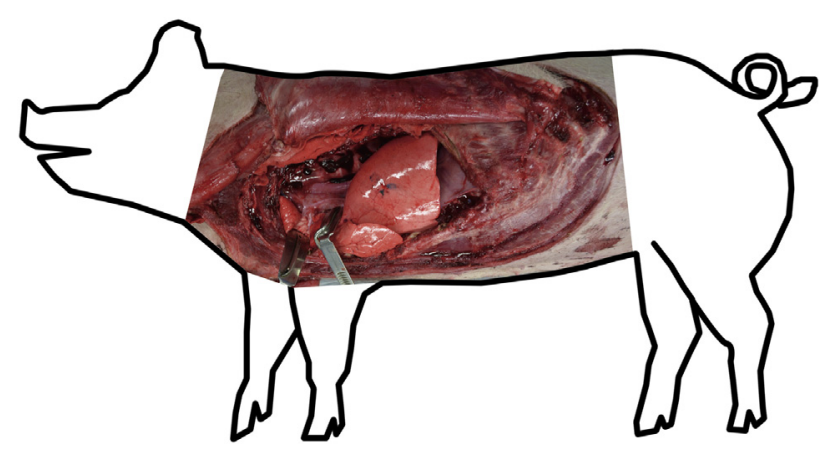

FIGURE 2. Schema of Yorkshire pig with surgical field image. Unilateral chest wall resection was performed to obtain the whole image of the thoracic cavity including lung and mediastinal structures.

\section{Dose Optimization of ICG and ICG:HSA In Vivo}

ICG and ICG:HSA exhibits intense quenching (ie, reduction of fluorescence emission) as its concentration is increased over a certain threshold. ${ }^{14}$ To determine the optimal concentration of ICG and ICG:HSA, we first measured the fluorescence intensities of ICG and ICG:HSA at various concentrations. The optimal dose of ICG or ICG:HSA in vivo was supposed to be different from the dose in vitro when the 2 fluorescents mixed with lymph fluid of body. ${ }^{18}$ Therefore, we tested the concentration-dependent fluorescent intensity in in vivo conditions. ICG and ICG:HSA at concentrations of $3.2,6.4,12.8$, and $32 \mu \mathrm{mol} / \mathrm{L}$ were injected into the rat footpad (Figure 3, A). The stained lymphatic vessels and nodes were observed at 30 minutes after injection using the ICFIS. We found that both ICG and ICG:HSA at $12.8 \mu \mathrm{mol} / \mathrm{L}$ provided the highest SBR in lymph nodes (Figure $3, B$ ). Considering the average weight of rats, the dosage of ICG is calculated as $5 \mu \mathrm{g} / \mathrm{kg}$.

\section{Comparison Between ICG Versus ICG:HSA}

In vivo and ex vivo NIR fluorescence images of lymph node were obtained at 5, 30, 60, and 120 minutes after injection with $5 \mu \mathrm{g} / \mathrm{kg}$ of ICG or ICG:HSA $(12.8 \mu \mathrm{mol} / \mathrm{L}$ in 0.1 $\mathrm{mL})$. The SLNs were identified in $10(100 \%) 10$ rats under in vivo conditions (Figure $4, A$ ). SBRs of the ICG and ICG:HSA mapped in lymph nodes were not significantly different in the 2 groups $(P=.28, .89, .15$, and .19 at times of 5, 30, 60, and 120 minutes) (Figure $4, B$ ), which showed similar fluorescence intensities in both groups.

To validate whether this system has a potential clinical use, we carried out preclinical studies with large animals. 


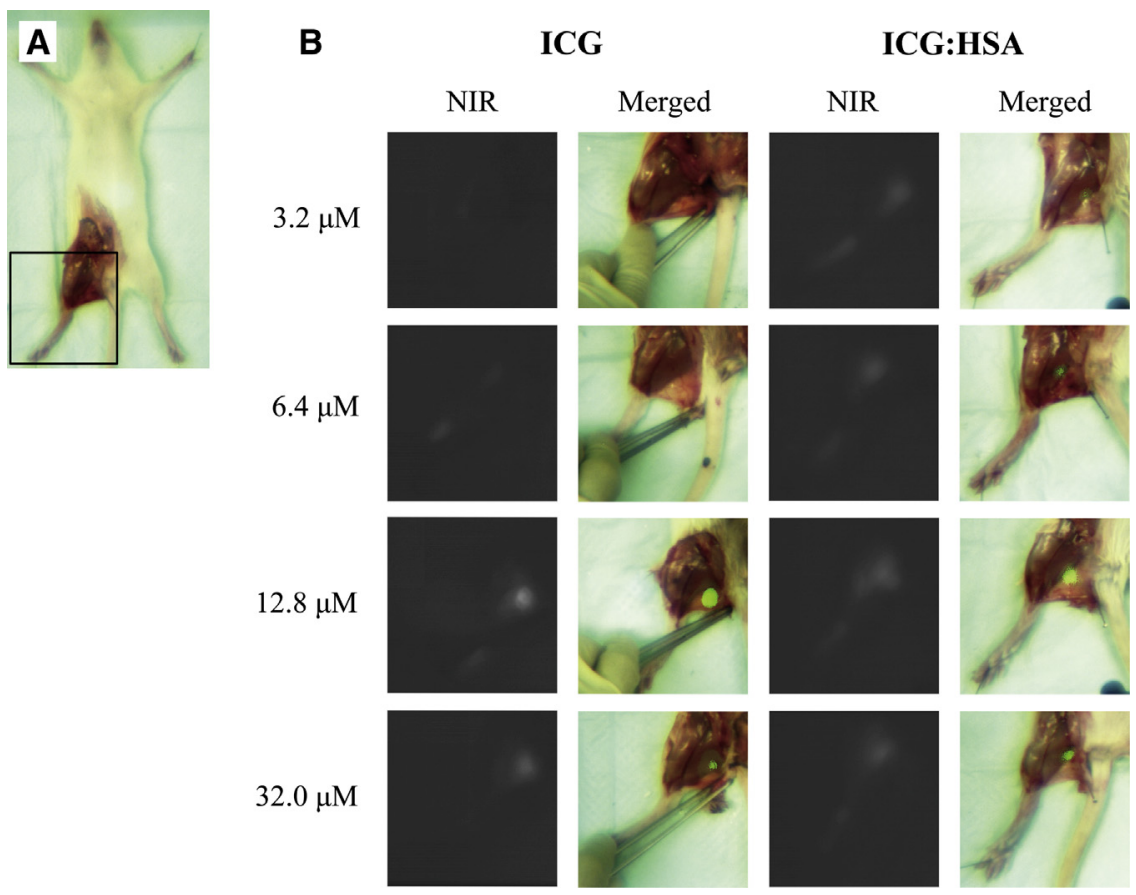

FIGURE 3. Optimization of ICG and ICG:HSA dose as a function of the complex trade-off between fluorescence quenching at the dilution of fluorophore in lymphatic channels. A, Right thigh (square) images of each rat were captured. B, Optimization of ICG and ICG:HSA mapped lymph node. A different dose of ICG and ICG:HSA ( 3.2 to $32 \mathrm{~mol} / \mathrm{L}$ ) was injected into the rat food pad. Each is displayed in NIR fluorescence images (left columns) and fusion images (right columns) using excitation light at $10 \mathrm{~mW} / \mathrm{cm}^{2}$. ICG, Indocyanine green; HSA, human serum albumin; NIR, near-infrared.

After opening the porcine chest, we injected $5 \mu \mathrm{g} / \mathrm{kg}$ of both fluorescents $(128 \mu \mathrm{mol} / \mathrm{L}$ in $1.5 \mathrm{~mL})$ at the lateral surface of the left upper lobe lung parenchyma in all pigs, a concentration consistent with the study in rats. In ICG cases, the SLNs were successfully visualized at the para-aortic area using ICFIS at around 7 minutes after injection. However, ICG:HSA was detected around 2 minutes later than ICG, consistent with other studies. ${ }^{19}$ All the SLNs were identified in $4(100 \%)$ pigs under in vivo conditions. ICG provided slightly higher total fluorescence yield in SLN than ICG:HSA (15.1 vs 7.2) (Figure 5). However, the SLN visibilities for both fluorescents were not significantly different.

\section{DISCUSSION}

Inasmuch as the world's population is aging, many thoracic surgeons are now faced with a growing number of patients with resectable lung cancer who have cardiopulmonary dysfunction or extensive comorbidities. ${ }^{11}$ The SLN biopsy is useful for the avoidance of unnecessary dissection of nonmetastatic nodes ${ }^{16}$ or determining the final indication for limited resection such as segmentectomy in those patients. ${ }^{20}$ SLN molecular studies could greatly improve the detection of micrometastases through more focused pathologic or molecular evaluation for staging. ${ }^{21}$ The staining of serial sections of SLNs by immunochemistry is the most frequently reported method of identifying micrometastases. Molecular analysis by reverse transcriptase-polymerase chain reaction offers a sensitive tool for the detection of micrometastasis. However, because the prognostic significance of molecular upstaging in lung cancer is not yet known, adjuvant chemotherapy has not been recommended in patients with micrometastases in lymph nodes. ${ }^{21}$ With increased experience, this technique may be useful in the management of early-stage lung cancer.

NIR fluorescence imaging for SLN mapping enables real-time visualization of SLN and lymphatic vessels. It has clear advantages over conventional methods such as blue dyes and radioisotopes owing to higher visibility in thick tissues and no radiation risk of NIR wavelength. ${ }^{7,13}$ A number of groups used NIR fluorescence imaging systems in preclinical and clinical studies. ${ }^{6,14,16,17,22}$ These devices were constructed either for open surgery or for thoracoscopic surgery.

As shown in Figure 1, we built an intraoperative color and fluorescence imaging system (ICFIS) for SLN detection in lung cancer surgery. Color and fluorescence merged images allowed real-time visualization of SLNs within the context of color surgical anatomy, enabling image-guided surgery without switching camera channels. Owing to real-time contrast control software, the dim fluorescence can be effectively displayed over the color images without switching or dimming the light in the operating room. ${ }^{6,23}$ For clinical translation, the excitation beam being delivered via the bifurcated optical fiber bundles needs to be replaced by 
ICG
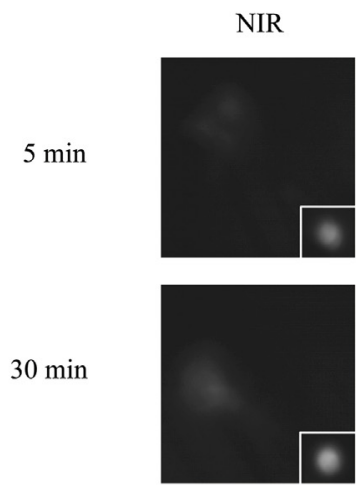

$60 \mathrm{~min}$

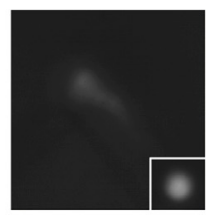

$120 \mathrm{~min}$

A

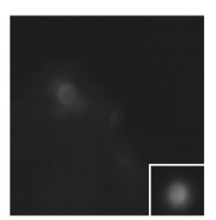

Merged
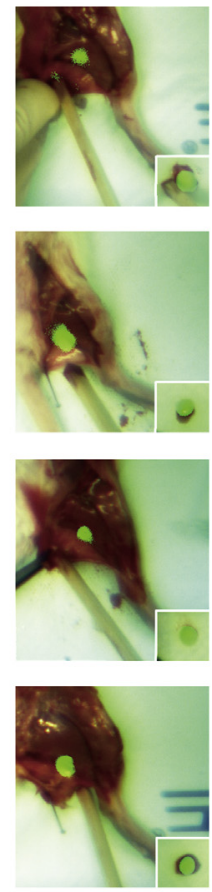

ICG:HSA
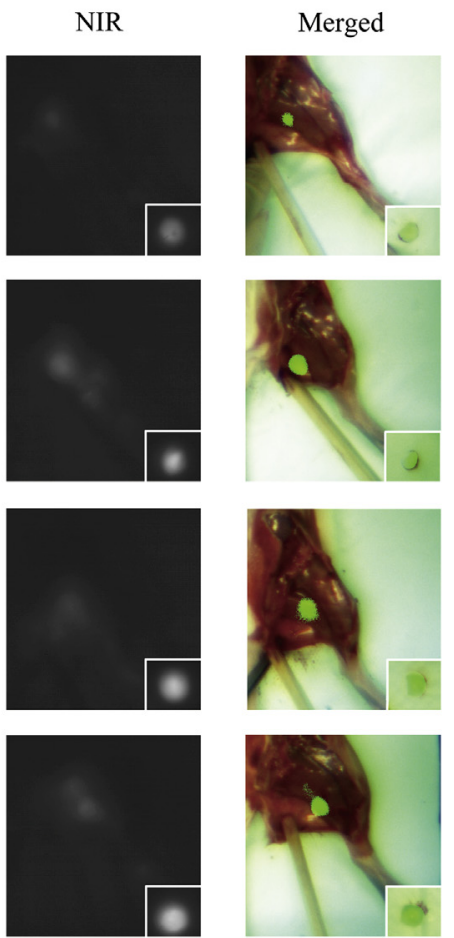

Comparison of In Vivo SLN

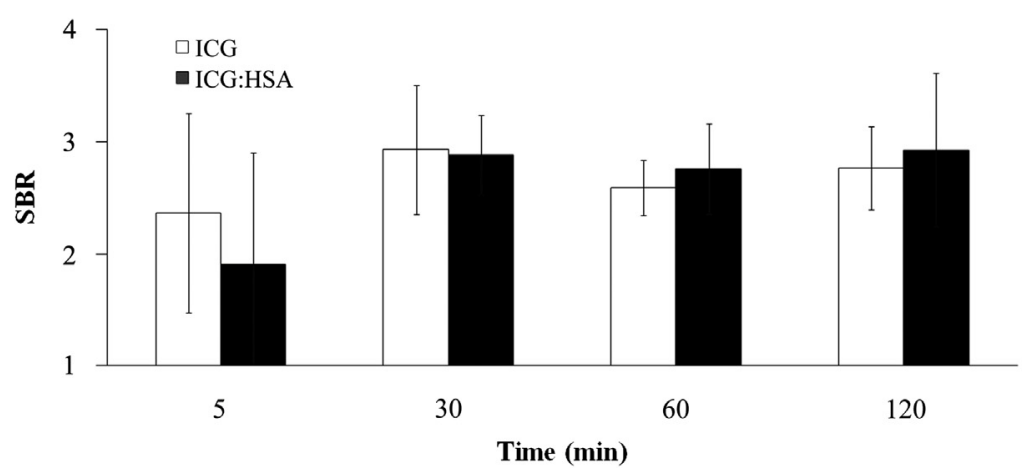

FIGURE 4. A, Comparison of ICG and ICG:HSA mapped lymph node NIR fluorescence images in the rat. A 0.1-mL dose of the ICG or ICG:HSA was injected into the food pad. Each is displayed in NIR fluorescence images (left columns) and fusion images (right columns) using excitation light at $10 \mathrm{~mW} / \mathrm{cm}^{2}$. Each is displayed in in vivo SLN images (big square) and ex vivo SLN images (small square). B, SBR of ICG and ICG:HSA for in vivo SLN mapping. SBR (mean $\pm \mathrm{SD}$ ) over time in the lymph nodes of rats $(\mathrm{n}=5$ independent animals for each tracer). ICG, Indocyanine green; $H S A$, human serum albumin; $N I R$, near-infrared; $S L N$, sentinel lymph node; $S B R$, signal to background ratio.

bright light-emitting diode sources for clinical safety. Except for this, the ICFIS has features similar to other fluorescence imaging systems that are useful for intraoperative procedures, and the results of SLN identification rate described in this study demonstrate the clinical feasibility of ICFIS in humans.

Recent trends for lung cancer surgery are minimally invasive approaches through video-assisted thoracoscopic surgery systems. Several NIR fluorescence thoracoscopic imaging systems connected with standard thoracoscopes have been introduced by other groups. However, commercially available thoracoscopes have poor transmission for the NIR wavelengths, delivering only a low portion of SBR fluorescence signals. To minimize this problem when ICFIS is finally coupled with the thoracoscopic systems, we will use a custom-built real-time contrast control software that would lower the least power of NIR excitation light necessary. Currently, we nearly manufactured the 


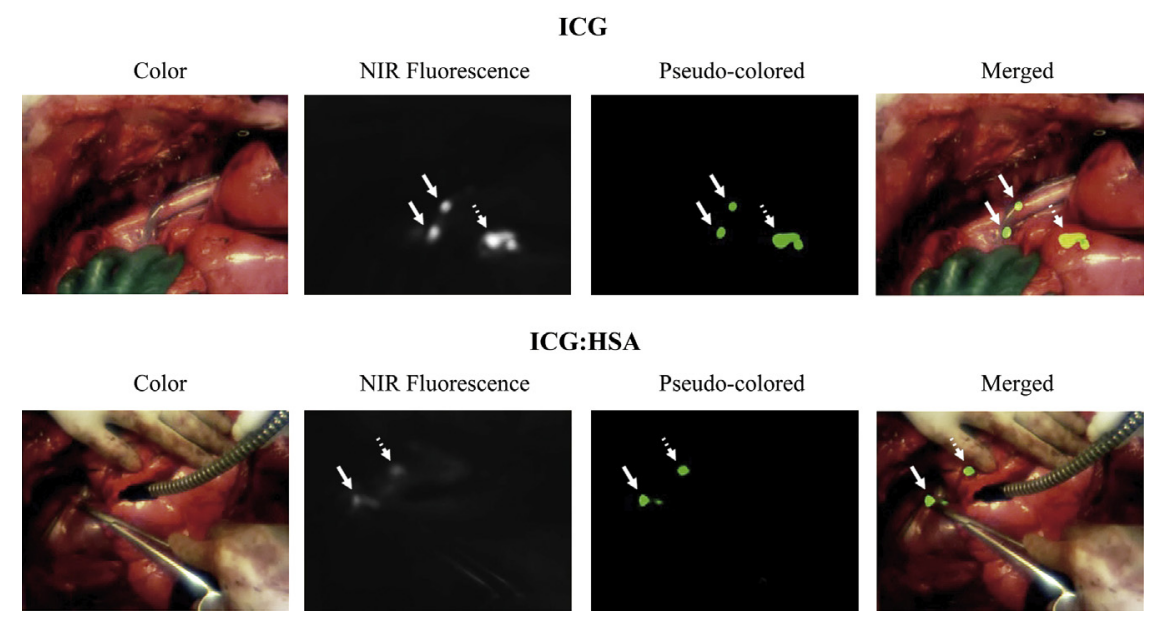

FIGURE 5. Comparison of ICG and ICG:HSA mapped lymph node NIR fluorescence images in preclinical trial. For each is displayed a color image (first column), a NIR-fluorescence image ( second column), contrast-enhanced and pseudo-colored image (third column), and a fusion image (fourth column) using excitation light at $5 \mathrm{~mW} / \mathrm{cm}^{2}$. Sentinel lymph nodes (arrows) and injected site (white dotted arrows) were visualized well with both tracers. ICG, Indocyanine green; $H S A$, human serum albumin; NIR, near-infrared.

thoracoscopic version of ICFIS, and the clinical feasibility may be validated in a future study.

Among various NIR fluorescence dyes for SLN mapping, ICG dyes are currently available dyes approved by the Food and Drug Administration. Accordingly, a number of preclinical and clinical studies using these dyes have been published. ${ }^{14,18}$ In addition, recent reports note that ICG:HSA (premixing ICG with HSA) increases the fluorescence intensity and the hydrodynamic diameter, giving better SLN retention with an improved detection rate over ICG alone. ${ }^{18}$ Our group has conducted a dose-finding study and demonstrated that the optimal concentration of ICG and ICG:HSA for NIR-based SLN mapping in animal studies lies in the range of $5 \mu \mathrm{g} / \mathrm{kg}$ (Figure 3). Even though our doses of ICG and ICG:HSA are significantly lower than those of other studies, ICFIS is sensitive enough to display the necessary signals in real time. ${ }^{14}$ We also submit that the dose higher than what we propose does not guarantee brighter fluorescence signals.

Comparing ICG with or without albumin premixing, our results imply that there is no significant difference in detecting SLN when the ICG concentrations in both solutions were identical. In the rat thigh, SBR of ICG and ICG:HSA were similar and remained constant from 30 to 120 minutes after injection (Figure 4). This result implies that ICG and ICG:HSA provided similar retention rates in the SLN. Although we did not get statistically significant results, in the porcine lung, ICG provided higher total fluorescence yield compared with ICG:HSA after 30 minutes (Figure 5). ICG is likely to flow more rapidly through narrow lymphatic channels, owing to its smaller hydrodynamic diameter than ICG:HSA, and get absorbed by the albumin abundant in lymph fluid. The longer traveling distance between the injection site and SLN could cause additional absorption of ICG to albumin in the lymph fluid. Inasmuch as porcine lung has longer lymphatic channels than rat thigh, the retention rate of ICG was slightly higher than ICG:HSA in porcine lung whereas the retention rate of both fluorophores was the same in rat thigh. Big animal studies were consistent with the clinical results conducted for breast cancer, in that ICG provided slightly higher total fluorescence yield than ICG:HSA at early times. ${ }^{14}$ From this study, for surgery for patients with lung cancer, injection at the primary tumor could cause still more increased traveling distance, aiding ICG absorption to albumin. As a result, ICG is likely to provide similar or better retention rate in SLN than ICG:HSA in lung cancer. These observations suggest the superiority of ICG as the currently available optimal fluorescent lymphotrophic tracer over ICG:HSA in lung cancer surgery, thereby reducing the cost and complexity of the procedure of premixing ICG with albumin. ${ }^{14}$

In conclusion, we developed an ICFIS for lung cancer surgery and performed a preclinical study of SLN mapping comparing ICG with or without albumin in the rat thigh and pig lung. Considering the anatomic structure of the lung, the ICG without premixing with albumin is considered to be enough fluorescent tracer inasmuch as it has little or no influence on the surgical outcome.

\section{References}

1. Ross MI. Sentinel node biopsy for melanoma: an update after two decades of experience. Semin Cutan Med Surg. 2010;29:238-48.

2. Little AG, DeHoyos A, Kirgan DM, Arcomano TR, Murray KD. Intraoperative lymphatic mapping for non-small cell lung cancer: the sentinel node technique. J Thorac Cardiovasc Surg. 1999;117:220-4.

3. Liptay MJ. Sentinel node mapping in lung cancer: the Holy Grail? Ann Thorac Surg. 2008;85:S778-9.

4. Khullar O, Frangioni JV, Grinstaff M, Colson YL. Image-guided sentinel lymph node mapping and nanotechnology-based nodal treatment in lung cancer using 
invisible near-infrared fluorescent light. Semin Thorac Cardiovasc Surg. 2009; 21:309-15.

5. Liptay MJ, Masters GA, Winchester DJ, Edelman BL, Garrido BJ, Hirschtritt TR, et al. Intraoperative radioisotope sentinel lymph node mapping in non-small cell lung cancer. Ann Thorac Surg. 2000;70:384-9.

6. Yamauchi K, Nagafuji H, Nakamura T, Sato T, Kohno N. Feasibility of ICG fluorescence-guided sentinel node biopsy in animal models using the HyperEye Medical System. Ann Surg Oncol. 2011;18:2042-7.

7. Liptay MJ, D'Amico TA, Nwogu C, Demmy TL, Wang XF, Gu L, et al. Intraoperative sentinel node mapping with technitium-99 in lung cancer: results of CALGB 140203 multicenter phase II trial. J Thorac Oncol. 2009;4:198-202.

8. Nomori H, Horio H, Naruke T, Orikasa H, Yamazaki K, Suemasu K. Use of technetium-99m tin colloid for sentinel lymph node identification in non-small cell lung cancer. J Thorac Cardiovasc Surg. 2002;124:486-92.

9. Melfi FM, Lucchi M, Davini F, Viti A, Fontanini G, Boldrini L, et al. Intraoperative sentinel lymph node mapping in stage I non-small cell lung cancer: detection of micrometastases by polymerase chain reaction. Eur J Cardiothorac Surg. 2008;34:181-6.

10. Rzyman W, Hagen OM, Dziadziuszko R, Kobierska-Gulida G, Karmolinski A, Lothe IM, et al. Intraoperative, radio-guided sentinel lymph node mapping in 110 nonsmall cell lung cancer patients. Ann Thorac Surg. 2006;82:237-42.

11. Kim S, Kim HK, Kang DY, Jeong JM, Choi YH. Intra-operative sentinel lymph node identification using a novel receptor-binding agent (technetium-99m neomannosyl human serum albumin, 99mTc-MSA) in stage I non-small cell lung cancer. Eur J Cardiothorac Surg. 2010;37:1450-6.

12. Kim H, Kim HK, Kang D, Lee DK, Choi YH, Lim SH. A comparative study of two- versus one-lung ventilation for needlescopic bleb resection. Eur Respir J. 2011;37:1183-8.

13. Polom K, Murawa D, Nowaczyk P, Rho YS, Murawa P. Breast cancer sentinel lymph node mapping using near infrared guided indocyanine green and indocyanine green-human serum albumin in comparison with gamma emitting radioactive colloid tracer. Eur J Surg Oncol. 2012;38:137-42.
14. Hutteman M, Mieog JS, van der Vorst JR, Liefers GJ, Putter H, Löwik CW, et al Randomized, double-blind comparison of indocyanine green with or withou albumin premixing for near-infrared fluorescence imaging of sentinel lymph nodes in breast cancer patients. Breast Cancer Res Treat. 2011;127:163-70.

15. Kim S, Lim YT, Soltesz EG, De Grand AM, Lee J, Nakayama A, et al. Near-infrared fluorescent type II quantum dots for sentinel lymph node mapping. Nat Biotechnol. 2003;22:93-7.

16. Yamashita S, Tokuishi K, Miyawaki M, Anami K, Moroga T, Takeno S, et al. Sentinel node navigation surgery by thoracoscopic fluorescence imaging system and molecular examination in non-small cell lung cancer. Ann Surg Oncol. 2012;19:728-33.

17. Yamashita S, Tokuishi K, Anami K, Miyawaki M, Moroga T, Kamei M, et al Video-assisted thoracoscopic indocyanine green fluorescence imaging system shows sentinel lymph nodes in non-small cell lung cancer. $J$ Thorac Cardiovasc Surg. 2011;141:141-4.

18. Ohnishi S, Lomnes SJ, Laurence RG, Gogbashian A, Mariani G, Frangioni JV. Organic alternatives to quantum dots for intraoperative near-infrared fluorescen sentinel lymph node mapping. Mol Imaging. 2005;4:172-81.

19. Mieog JS, Troyan SL, Hutteman M, Donohoe KJ, van der Vorst JR, Stockdale A et al. Toward optimization of imaging system and lymphatic tracer for nearinfrared fluorescent sentinel lymph node mapping in breast cancer. Ann Surg Oncol. 2011;18:2483-91.

20. Nomori H, Kohno M, Izumi Y, Ohtsuka T, Asakura K, Nakayama T. Sentinel nodes in lung cancer: review of our 10-year experience. Surg Today. 2011;41:889-95.

21. Melfi FM, Davini F, Boni G, Mussi A. Sentinel lymph node in lung cancer surgery. Thorac Surg Clin. 2012;22:205-14.

22. Schaafsma BE, Mieog JS, Hutteman M, van der Vorst JR, Kuppen PJ, Löwik CW et al. The clinical use of indocyanine green as a near-infrared fluorescent contrast agent for image-guided oncologic surgery. J Surg Oncol. 2011;104:323-32.

23. Troyan SL, Kianzad V, Gibbs-Strauss SL, Gioux S, Matsui A, Oketokoun R, et al. The FLARE intraoperative near-infrared fluorescence imaging system: a first-inhuman clinical trial in breast cancer sentinel lymph node mapping. Ann Surg Oncol. 2009;16:2943-52. 